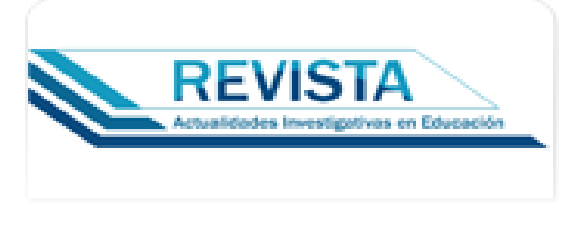

\author{
Revista Electrónica "Actualidades \\ Investigativas en Educación" \\ E-ISSN: 1409-4703 \\ revista@inie.ucr.ac.cr \\ Universidad de Costa Rica \\ Costa Rica
}

Artavia Granados, Jenny María

MANIFESTACIONES DE VIOLENCIA EXPLÍCITA O EVIDENTE DURANTE EL DESARROLLO DEL

RECREO ESCOLAR

Revista Electrónica "Actualidades Investigativas en Educación", vol. 12, núm. 2, mayo-agosto, 2012,

pp. 1-29

Universidad de Costa Rica

San Pedro de Montes de Oca, Costa Rica

Disponible en: http://www.redalyc.org/articulo.oa?id=44723437001

- Cómo citar el artículo

Número completo

- Más información del artículo

- Página de la revista en redalyc.org

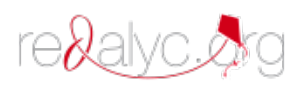

Sistema de Información Científica

Red de Revistas Científicas de América Latina, el Caribe, España y Portugal

Proyecto académico sin fines de lucro, desarrollado bajo la iniciativa de acceso abierto 


\title{
MANIFESTACIONES DE VIOLENCIA EXPLÍCITA O EVIDENTE DURANTE EL DESARROLLO DEL RECREO ESCOLAR
}

MANIFESTATIONS OF EXPLICIT OR IMPLICIT VIOLENCE

DURING OF THE SCHOOL RECESS

\author{
Volumen 12, Número 2 \\ Mayo-Agosto \\ pp. 1-29
}

Este número se publicó el 30 de mayo de 2012

Jenny María Artavia Granados

Revista indizada en REDALYC

Revista distribuida en las bases de datos:

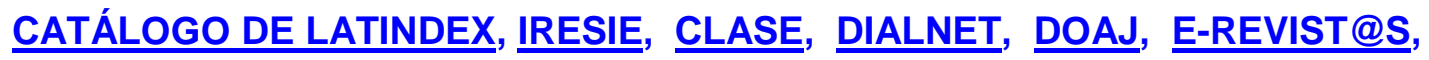

Revista registrada en los directorios:

ULRICH'S, REDIE, RINACE, OEI, MAESTROTECA, PREAL, HUASCARAN, $\underline{\text { CLASCO }}$ 


\title{
MANIFESTACIONES DE VIOLENCIA EXPLÍCITA O EVIDENTE DURANTE EL DESARROLLO DEL RECREO ESCOLAR MANIFESTATIONS OF EXPLICIT OR IMPLICIT VIOLENCE DURING OF THE SCHOOL RECESS
}

\begin{abstract}
Jenny María Artavia Granados ${ }^{1}$
Resumen: Este artículo es producto de un proceso de investigación realizado en una escuela de la Dirección Regional de Educación Occidente. Su finalidad fue determinar si en el intervalo de los recreos de la escuela en estudio, se suscitaban manifestaciones de violencia escolar explícita entre estudiantes de IV, V y VI año del II Ciclo de la Educación General Básica. Durante el desarrollo del estudio, se tomaron en cuenta aspectos relacionados con las actividades lúdicas y las interacciones personales que se presentaban en estos espacios escolares. Sobre todo, los que podrían propiciar violencia escolar explícita; los mecanismos de prevención y estrategias empleadas por la escuela para contrarrestar la violencia escolar; también el nivel de integración del personal docente y administrativo de la escuela durante el periodo de los recreos. Asimismo, se plantearon algunas conclusiones entre las que destacan que un alto porcentaje de niños y niñas manifiesta que existen enfrentamientos entre estudiantes, durante el desarrollo de los recreos, y que estos se perciben a través de comportamientos de violencia tanto física como verbal. Las manifestaciones de violencia más comunes son peleas, golpes así como el uso de un lenguaje soez y ofensivo. Los motivos de los enfrentamientos varían según el nivel escolar entre el estudiantado. Los mecanismos empleados por la escuela para contrarrestar la violencia que se suscita durante los recreos, se basan en el uso de procedimientos sancionadores y la aplicación de medidas preventivas.
\end{abstract}

Palabras clave: RECREO ESCOLAR, VIOLENCIA ESCOLAR DURANTE LOS RECREOS, EDUCACIÓN PRIMARIA, EDUCACIÓN GENERAL BÁSICA, COSTA RICA.

Abstract: This article is the result of a research conducted in a primary school of Dirección Regional de Educación de Occidente. Its main goal is to determine whether violent behaviors are shown among fourth, fifth, and sixth graders in II Cycle of Educación General Básica during school recesses. During this study aspects dealing with personal interaction and ludic activities exhibited during recesses were analyzed especially those that showed explicit violence. At the same time, the strategies and prevention programs developed by the school as well as the participation of administrative and teaching personnel in recesses were studied. Lastly, some conclusions suggest that a high percentage of the population studied believe there are confrontations among students showings physical and oral violence during recesses which are shown by the physical and verbal behaviors displayed. The most common violent behaviors are fights, hits, and inappropriate language. Some of the mechanisms applied by the school administrators to minimize violence are sanctions and prevention measures.

Key words: RECESSES, SCHOOL VIOLENCE, SCHOOL VIOLENCE DURING RECESSES, EXPLICIT VIOLENCE DEMONSTRATIONS DURING RECESSES.

\footnotetext{
1 Doctora en Educación de la Universidad Estatal a Distancia, Máster en Psicopedagogía de la Universidad Estatal a Distancia, Licenciada en Educación Primaria y en Adminstración Educativa de la Universidad de Costa Rica. Docente del Departamento Ciencias de la Educación en la Sede de Occidente de la Universidad de Costa Rica.
}

Dirección electrónica: jargra@gmail.com

Artículo recibido: 10 de enero, 2012

Aprobado: 12 de abril, 2012 


\section{Introducción}

El término violencia proviene del latín violentia, o sea fuerza. Según la Organización Mundial de la Salud (2001), se entiende por violencia, aquella manera de obrar acompañada de coacción o fuerza física, sobre una persona, a fin de influir en su voluntad y obligarla a actuar de manera determinada. La violencia equivale a obligar a una persona, haciendo uso de la fuerza, la intimidación o la coacción moral, a obrar contra su voluntad.

Vinyamata (2003, p. 73), opina que "la violencia es todo aquello que puede representar o significar perjuicio, producir por efecto o defecto un mal a otro, a uno mismo o al entorno, ya sea realizado o llevado a cabo de manera consciente o inconsciente". Por su parte, Abramovay (2005) considera que en todas las definiciones del concepto de violencia, existe el consenso, que, "todo y cualquier acto de agresión física, moral o institucional dirigido contra la integridad de uno o varios individuos o grupos, es considerado como acto de violencia" (2005, p. 16).

En el estudio que dio lugar a este artículo, se asumió el concepto de violencia explícita como aquel o aquellos comportamientos mediante los cuales una o un estudiante, o grupo de estudiantes emplean la palabra o la fuerza física sobre otro u otros, con la finalidad de causar daño o maltrato físico, emocional o moral.

\section{Violencia en el contexto escolar}

La violencia que se desarrolla en las escuelas está determinada básicamente por dos factores: en primera instancia, se encuentran los factores externos a la institución, como son aquellos de índole social y familiar, que penetran a la institución escolar. Según Brawer (2006) la violencia en la escuela, se refiere a los hechos que tienen a la escuela como escenario, pero que no son producto de las prácticas que en ella se desarrollan.

Por otra parte, se encuentra la existencia de la violencia escolar, la cual se produce en la institución educativa, producto de procesos antidemocráticos provocados por el propio sistema educativo. Entre ellos se puede citar: el empleo de metodologías autoritarias, las luchas de poder entre estudiantes, docentes y personal administrativo, el elevado número de estudiantes que impide la personalización de la tarea educativa y la falsa moralidad que ha generado discriminaciones de raza, culto, género y de capacidades. Según Osorio (2006, p.77) la violencia escolar o de la escuela, se define como "aquellos episodios de hostilidad, de agresión, de maltrato, de discriminación que genera el sistema educativo". 
Para Osorio (2006) el término de violencia escolar, está supeditado a una base ideológica construida socialmente bajo las ideologías de los gobernantes, cuya finalidad es explicar el fenómeno de la violencia que se manifiesta en las instituciones educativas, obviando la situación social y económica en que viven niños y jóvenes en las diferentes comunidades. Desde esta perspectiva se procura atribuir a otros entes sociales como la familia, la escuela, entre otros, la responsabilidad total del desarrollo del fenómeno de la violencia, dejando del lado la cuota de responsabilidad que tienen las instancias gubernamentales.

\section{¿Cuáles son las manifestaciones de la violencia en la escuela?}

La violencia que viven niños y jóvenes en sus hogares y comunidades, generalmente es provocada por la problemática social y económica en que están sumergidos, pero, también mucha de esta violencia es asimilada por medio de la televisión, el cine o los juegos electrónicos, así como la que se genera en el interior de la misma escuela.

El aumento de la violencia se ha convertido en un fenómeno manifiesto tanto en forma explícita, como en forma oculta en los salones de clase, en los pasillos y en el patio escolar. Estas manifestaciones hostiles van desde la burla, la ridiculización, el aislamiento, hasta la agresión física. Esto torna al centro educativo como un lugar inseguro y problemático. Según Abad (2002), las agresiones a los docentes han aumentado y éstas, se manifiestan de manera física y verbal. Asimismo, se presentan acciones violentas que procuran dañar la infraestructura escolar, el mobiliario y los materiales didácticos de las instituciones educativas.

En el interior de las instituciones educativas, se presentan algunas manifestaciones de violencia, entre las cuales se citan,

\section{- Disrupción en las aulas}

La disrupción en las aulas se da cuando algunos estudiantes no permiten con su comportamiento inadecuado, el desarrollo normal de las lecciones. Esta situación hace que el docente se vea obligado a emplear su tiempo en controlar la disciplina y el orden.

\section{- Indisciplina}

Los problemas de disciplina que se generan entre las y los estudiantes y el docente se convierten en problemas de violencia que pueden ir desde un grado menor hasta un alto grado. Martínez-Otero (2005) señala que en los salones de clase se producen problemas 
que van desde la resistencia pasiva hasta el desafío y el insulto hacia el docente. La indisciplina puede provocar agresiones, especialmente verbales, las cuales pueden ocasionarse desde el estudiante al profesor y viceversa.

\section{- Violencia física, verbal y psicológica}

El fenómeno de la violencia que se presenta en las instituciones escolares tiene como fin el lastimar al otro, ya se de forma física, psicológica, verbal o moral.

Para Olweus citado por Ortega (2004, p. 2), "el maltrato entre iguales se ha descrito como un comportamiento prolongado de insulto verbal, rechazo social, intimidación psicológica y lo agresividad física de unos niños hacia otros que se convierten, de esta forma, en víctimas de sus compañeros".

Desde esta perspectiva, la violencia que se suscita en los centros educativos, producen manifestaciones, entre las que se citan,

\section{Violencia Física:}

En el fenómeno de la violencia física, la escuela experimenta episodios de violencia externa que pretenden producir daño. Estos incidentes de violencia se producen de forma directa e indirecta. Desde la perspectiva de la violencia directa, el agresor actúa directamente sobre la víctima, propinándole patadas, empujones, puñetazos, zancadillas, entre otros actos. En la violencia indirecta, el agresor actúa sobre las pertenencias de la persona víctima de su agresión o causa daños a la infraestructura de la institución. Algunos de los actos de violencia indirecta que se pueden suscitar son: los robos, destrozos a los recursos didácticos o infraestructura, o bien, esconder las pertenencias de la víctima.

\section{Violencia verbal:}

A través de la violencia verbal, el agresor acosa a su víctima a través de los insultos, apodos o hablar mal de ésta. Según Casals (2006, p. 47), "los motes y los insultos son los dos tipos de abuso más frecuentes, en esto no hay diferencias de género que valgan y se dan con las demás formas de acoso". Este tipo de violencia resulta ser menos perceptible porque no existe una evidencia física del maltrato, pero se ejerce violencia a través del lenguaje. Las palabras empleadas por las y los niños, expresan insultos y humillaciones a sus pares, con los cuales generan un alto grado de violencia. 


\section{Violencia psicológica:}

La violencia psicológica o emocional, es un tipo de violencia que no es evidente y se produce de forma muy sutil. Es una forma de violencia que se manifiesta por medio de agresiones verbales, gestos, intimidaciones, maltrato y amenazas, entre otras.

La violencia psicológica fomenta comportamientos que llevan a una persona o grupo de personas a ignorar; no dejar participar; rechazar o amenazar a otro u otros. A través de este tipo de maltrato, se procura infundir temor a la víctima.

\section{- La intimidación}

El proceso de intimidación y victimización entre iguales es un fenómeno que se presenta comúnmente en las escuelas y colegios. A este tipo de violencia se le denomina también como bullying. Según Nogueira (2005), el bullying escolar es un término que se emplea para designar el maltrato y la intimidación entre los pares. Este fenómeno se presenta como un malestar que se manifiesta de manera oculta.

Los estudiantes que acosan e intimidan a otro (víctima), generalmente lo hacen a través de insultos, rumores, apodos, aislamiento social, etc. Este tipo de situaciones puede provocar en la víctima bajo rendimiento académico, fobia de asistir al centro educativo, tristeza o depresión y pérdida de autoestima. Al respecto, Nogueira (2005) aduce que las víctimas de la violencia entre pares, son frágiles; se sienten desiguales; perjudicados y generalmente no piden ayudan. Asimismo, estos tienen miedo de ir a la escuela, pueden presentar síntomas depresivos, pierden el sueño y se muestran dispersos en clase.

\section{- Violencia sexual}

El acoso sexual es un fenómeno o manifestación oculta de comportamiento antisocial. Este tipo de violencia se da cuando la persona asume el poder con que cuenta como un instrumento para el control y la dominación de otro. Se le hostiga mediante actos con contenido sexual. Tal y como lo indica, Martínez-Otero (2005, p. 98) "la agresión sexual es un tipo de violencia invisible, que sufre más la población femenina que la masculina, y que, por lo común, se concreta en hostigamientos, en tocamientos, y, en menor medida, en violaciones".

\section{- Violencia contra la escuela}

La violencia contra la escuela es un fenómeno mediante el cual niños y jóvenes producen daños materiales, a través del vandalismo, a las instalaciones físicas de escuelas y colegios y sobre los materiales didácticos o mobiliario. 


\section{- Violencia estudiantil interinstitucional}

La violencia estudiantil interinstitucional se lleva a cabo cuando se dan confrontaciones violentas entre estudiantes de diferentes centros de educación, especialmente de educación secundaria.

Las rivalidades entre instituciones surgen a partir de las comparaciones que se realizan de aspectos deportivos o académicos. Estas rivalidades pueden llegar a convertirlos en enemigos en un ámbito más generalizado y provocar serios problemas estudiantiles.

\section{Metodología empleada en la investigación}

Este artículo es producto de una investigación de corte cuantitativa, con un alcance que corresponde a un estudio descriptivo, realizada por Artavia (2010). En este estudio se analiza la violencia escolar explícita e implícita en las y los estudiantes de segundo ciclo de una escuela pública de la Dirección Regional de Educación Occidente, durante el desarrollo de los recreos.

Para recolectar los datos se realizaron veinte observaciones del desarrollo de los recreos. Se empleó una guía estructurada de observación en la cual se entrevistaron catorce docentes y la Directora de la escuela en estudio. Además, se pasaron cuestionarios a las y los estudiantes sujetos del estudio.

La población total de niños y niñas de estos niveles escolares está constituida por trescientos cuarenta y cinco estudiantes, distribuidos en cinco secciones de IV año, cuatro secciones de V año y cinco secciones de VI año. El cuestionario se aplicó a ciento ochenta estudiantes del segundo ciclo, elegidos al azar. Fue contestado por sesenta niños y niñas de cada nivel. De la población estudiantil elegida, devolvieron el cuestionario ciento cincuenta estudiantes, representando el 52\% de la población total, del segundo ciclo.

Estas técnicas se emplearon con la finalidad de recoger información general sobre los recreos y las posibles conductas violentas que desarrollan los y las niñas durante el desarrollo de los recreos. Para garantizar la confidencialidad y anonimato de los datos se procedió a identificar a los sujetos del estudio con diferentes letras del abecedario.

Los ejes que guiaron el proceso de investigación durante el desarrollo de los recreos fueron:

a) Interacciones personales que se suscitan en las actividades lúdicas que comparten los estudiantes. 
b) Conductas violentas explícitas e implícitas de las y los estudiantes durante el recreo.

c) Mecanismos empleados por estudiantes y docentes en la prevención de conductas violentas.

d) Estrategias empleadas por el personal docente y administrativo para contrarrestar las conductas violentas.

e) Nivel de integración del personal docente y administrativo de la escuela, durante el desarrollo de los recreos.

\section{Manifestaciones de violencia explícita o manifiesta durante el desarrollo del recreo}

A través de las observaciones, entrevistas y los cuestionarios aplicados a los sujetos del estudio, fue posible obtener información importante respecto a las manifestaciones de violencia explícita o manifiesta, en el desarrollo de los recreos de la escuela, en estudio. Se presentan a continuación las opiniones expresadas por las docentes, Directora de la institución y estudiantes de la escuela que sirvieron de base para la investigación realizada por Artavia (2010).

\section{Conductas violentas explícitas de las y los estudiantes durante el recreo}

En las conductas de violencia explícita un grupo de estudiantes emplea manifestaciones de violencia con la palabra o la fuerza física (golpes, empujones, patadas, entre otros), para causar daño o maltrato físico al otro.

Seguidamente, se expone el análisis de la información concerniente a aquellas conductas de violencia explícita, observadas durante el desarrollo de los recreos en la escuela en estudio. Asimismo, se presenta información suministrada producto de los cuestionarios y las entrevistas, realizadas a estudiantes, docentes y la Directora de la institución. 


\section{Gráfico $\mathbf{N}^{\circ} 1$}

Existencia de enfrentamientos entre estudiantes, durante los recreos en la escuela en estudio. Año 2010

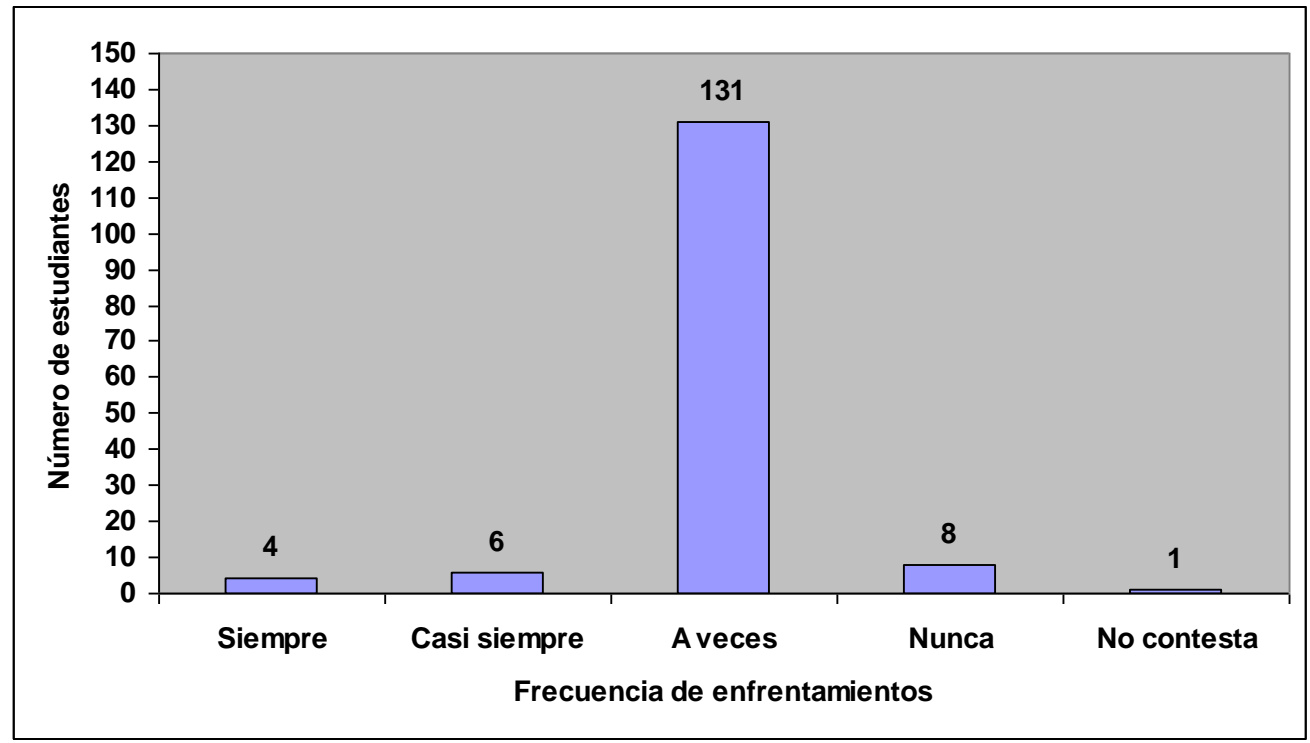

Fuente: Artavia, Jenny (2010). Resultados del cuestionario aplicado a estudiantes II Ciclo Enseñanza General Básica, escuela en estudio.

El gráfico No 1 suministra información relacionada con la pregunta realizada a las y los estudiantes, acerca de la frecuencia con que se producen enfrentamientos o peleas entre ellos, durante el desarrollo del recreo escolar. Según los resultados obtenidos, se evidencia que 131 (87 por ciento) de las y los estudiantes manifiestan que a veces se producen enfrentamientos en los recreos, ocho ( 5 por ciento) que nunca, seis ( 4 por ciento) casi siempre y cuatro (2,5 por ciento) siempre. El tipo de enfrentamiento donde la manifestación de violencia es explícita, es fácil de visualizar en el patio de juegos a través de empujones, zancadillas, golpes que se producen durante los juegos que se desarrollan.

Durante las observaciones realizadas se pudo constatar que en los recreos se producen enfrentamientos o peleas entre estudiantes. Estos se suscitan durante el transcurso de las actividades lúdicas y en las interacciones personales en las que participan las y los estudiantes sujetos del estudio. 
Las niñas y niños practican juegos como ${ }^{2}$ la anda, ${ }^{3}$ quedó congelado, futbol, ${ }^{4}$ ladrones y policías, en donde se empujan unos contra otros, se dan golpes y alguna que otra vez, un niño le pone la zancadilla a otro que va corriendo rápidamente. A continuación se cita una serie de ejemplos de observaciones realizadas en el patio escolar durante el desarrollo de los recreos.

- Un grupo de niños juega futbol. Al tratar de quitarle la bola al otro equipo, los niños se tornan violentos. Se patean y empujan, sus caras cambian y evidencian un gran enojo. (Observación día 25-9-08)

- En uno de los recreos, una niña estaba sentada en un muro de uno de los pasillos de la escuela que conduce al gimnasio. Los varones pasaban corriendo rápidamente. Cada vez que alguno venía corriendo ella alzaba la pierna para darle una zancadilla. (Observación día 8-10-08)

- Un estudiante corre velozmente por uno de los pasillos, empuja a una niña, ésta cae, se golpea fuertemente la nariz y le provoca sangrado. (Observación día 11-10-08)

Al cuestionar a las docentes acerca de si, ¿consideran que en la escuela existe violencia escolar? Estas adujeron que,

- Sí, de diferentes tipos: desde la verbal, hasta física donde se les ve que meten la zancadilla a otro niño. Pero, sí hay violencia y se muestra en el enojo de los niños y cómo logran manejar las emociones." (Docente E de VI año, 22 de setiembre 2009).

- En algunos niños sí, son muy bruscos para jugar y los juegos no son los adecuados para compartir con sus compañeros." (Docente C de IV año, 22 de setiembre 2009).

- En algunas ocasiones yo he visto golpes quizás no muchos, ni en mi grupo, pero, sí he visto puñetazos que un niño le da a otros o he visto darse de patadas hasta niños

\footnotetext{
${ }^{2}$ La anda es un juego donde un niño designado por sus compañeros, debe correr y tratar de alcanzar a alguno de ellos y tocarlo, inmediatamente quien lo tocó grita: "la anda" para que todos se enteren de que ahora se deben cuidar de no ser tocados por ese compañero.

${ }^{3}$ El quedó congelado es otro juego donde un compañero es designado para que corra tras sus pares y trate de tocarlos, para que estos queden congelados. Una vez congelado, no se podrá mover hasta que otro compañero pase y lo toque, y así se descongele y siga jugando.

${ }^{4}$ En el juego de ladrones y policías, el grupo de niños se divide en dos: unos asumen el rol de policías y otros de ladrones. Los policías deben procurar atrapar a los ladrones que corren veloces, una vez que se atrapa a un ladrón este es llevado a un lugar llamado la cárcel, que es resguardado por un policía para que no se puedan salir los encarcelados. En este juego gana el equipo que en el caso de los policías logra atrapar a la mayor cantidad de ladrones o en el caso de los ladrones, si la mayoría de los participantes no son atrapados por los policías.
} 
contra niñas. Esos eventos yo antes, no los había visto". (Docente A de V año, 23 de setiembre 2009)

- Sí claro, cuando juegan quedó congelado pero brusco. Como que se vienen a quitar un montón de frustraciones aquí a la escuela, pegándole violentamente a otros. Yo los he visto en juegos que tienen otros nombres, porque ya ellos les cambiaron los nombres según el golpe, pero no sé el nombre. Cuando juegan policías y ladrones son capaces de tomar a otros niños y lastimarlos". (Docente I de V año, 23 de setiembre 2009).

Por otra parte, la Directora de la institución considera que sí existe en la institución violencia. Al respecto manifiesta:

- Sí, sí hay violencia escolar porque los niños vienen a sumar todo lo que viven en sus hogares. En los hogares están viviendo una sociedad muy violenta porque hay un factor que está influyendo en que el alimento diario del niño, sea violencia. (26 de noviembre 2009).

Asimismo, en los recreos de la escuela en estudio, la violencia verbal está también presente. Es frecuente escuchar a niños y niñas emplear un vocabulario soez contra sus compañeros. Es común escuchar el empleo de palabras como "hijo de puta", "estúpido", "mal parido", "idiota”, "playo", "cara de picha”, entre otras palabras, las cuales son empleadas para insultar y molestar al otro.

Algunos de las y los estudiantes anotaron en el cuestionario algunas otras palabras y frases que emplean para insultar a los otros. Entre ellas, se citan:

- "Prostitutas vayan a comer caca, mamadoras. Solo por amistad que uno anda de la mano le dicen: son novias, son marimachas".

- "Ramera, zorra, bruta, zorra esquinera, que se paran en una esquina acostarse con los maes".

La Directora considera que existe en la escuela durante los recreos violencia verbal. Al respecto, señala: "Ahora es muy común ver niños que tienen un vocabulario destructivo hacia el ser humano, hacia la sociedad, hacia la persona que es figura e imagen del Señor. También hay violencia emocional, hay niños que son felices al humillar y hacer sentir mal a otros."

Las docentes también hicieron referencia de la presencia en los recreos de manifestaciones de violencia verbal, con el propósito de ofender y humillar a los otros. 


\section{Gráfico № 2}

Frecuencia con la que se presentan enfrentamientos

o problemas entre estudiantes según sexo, en los

recreos de la escuela en estudio. Año 2010

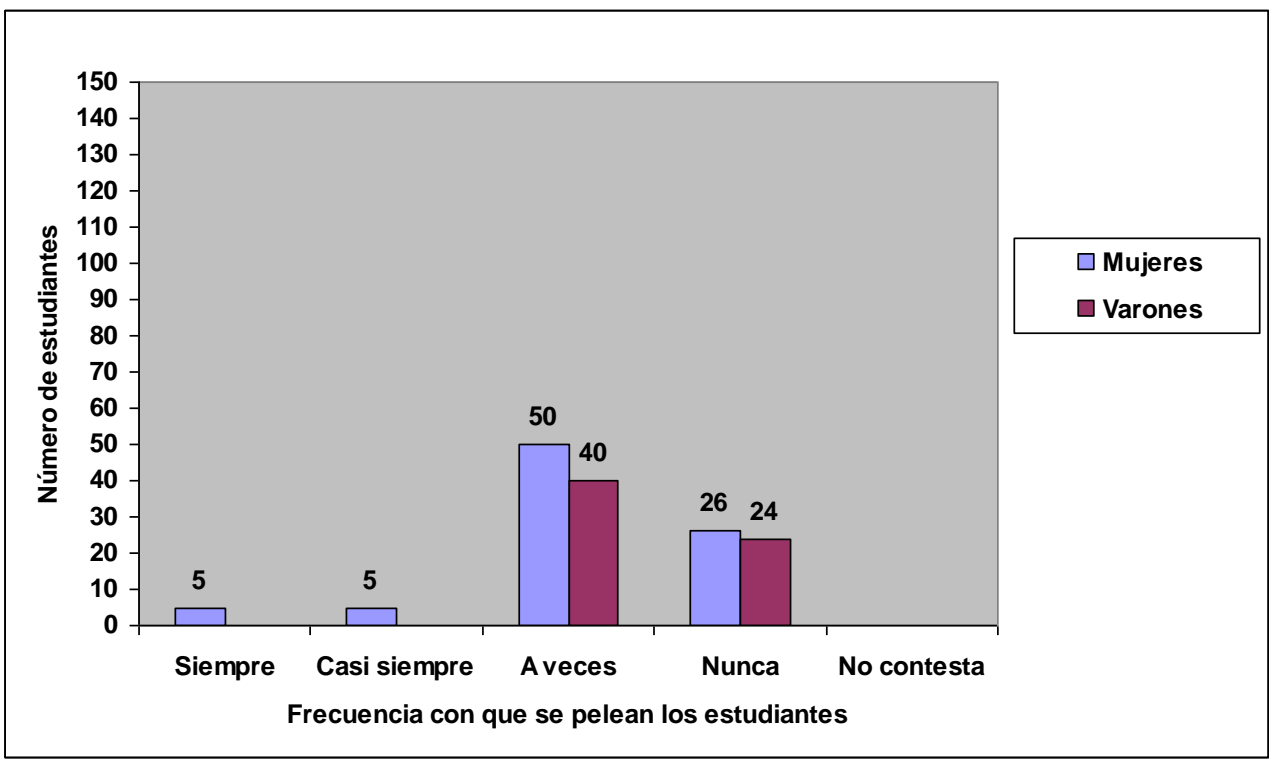

Fuente: Artavia, Jenny (2010). Resultados del cuestionario aplicado a estudiantes II Ciclo Enseñanza General Básica, escuela en estudio.

El gráfico No 2 permite observar la frecuencia con que se presentan enfrentamientos o problemas entre estudiantes según sexo, durante el desarrollo de los recreos. Según el género de los estudiantes, 50 (58 por ciento) de las mujeres considera que a veces se dan este tipo de problemas. Por su parte, 40 (63 por ciento) de los varones encuestados, considera que a veces hay enfrentamientos entre compañeros. Asimismo, 26 niñas (30 por ciento) opinan que nunca se producen enfrentamientos y 24 niños (37 por ciento) también señalan que nunca se producen enfrentamientos. Por otra, parte cinco mujeres (6 por ciento) señalan que siempre y casi siempre se producen los enfrentamientos.

En el cuestionario se les preguntan los motivos por los cuales se pelean. Sus respuestas permiten evidenciar que éstas se producen por diversas causas, entre ellas se citan:

\section{$\underline{\text { Respuestas de los estudiantes de IV año }}$}

- "Porque se tocan la cara".

- "Por reputación, o por molestar".

- "Porque dicen muchos apodos". 


\section{Respuestas de los estudiantes de $\mathrm{V}$ año}

- "Porque juegan muy brusco y se golpean sin culpa y el otro piensa que es con culpa".

- "Porque alguno le quita algo a otro; uno insulta al otro".

- "Porque a veces a uno le quitan la novia".

\section{$\underline{\text { Respuestas de los estudiantes de VI año }}$}

- "Por muchos insultos".

- "Porque tienen algún problema, porque se dicen cosas".

- "A veces por chiquillas o por insulto

Revisando los motivos que apuntan las y los niños por los cuales se presentan enfrentamientos entre estudiantes, puede visualizarse que para los estudiantes de cuarto año, sus motivos difieren bastante de los chicos y chicas de quinto y sexto año. Los y las niñas de IV año se enfrentan con sus pares como resultado de los juegos que comparten o por violencia verbal. Mientras, que los grados superiores ( $\mathrm{V}$ y $\mathrm{VI}$ año) las y los estudiantes se enfrentan entre sí, por asuntos relacionados con la percepción que tienen de los otros, por viejos problemas o rencillas personales y además por aspectos que tienen que ver con relaciones sentimentales que mantienen con personas del otro sexo.

En la entrevista con la Directora, esta afirma que "se da mucha violencia verbal y física. Hay muchos que juegan a base de golpes, se dan retos, pero es el reto para golpear, para hacer ver quién es el ganador". (26 de noviembre 2009)

Las docentes también opinan que es frecuente observar niños que durante el recreo expresan manifestaciones de violencia. Al respecto se citan algunas de sus opiniones:

- "Sí, muchas veces la misma rivalidad entre los niños fomenta para que den pie a la agresión entre otros niños". (Docente V de IV año, 22 de setiembre 2009).

- "A veces se logran ver niños en los recreos que agreden a otros de forma física y verbal, son tan rápidos en las acciones, que a uno se le dificulta encontrarlos en el momento oportuno". (Docente A, V año, 23 de setiembre 2009). 


\section{Mecanismos empleados por estudiantes y docentes en la prevención de conductas violentas}

La violencia es una problemática que requiere de un abordaje preventivo, en donde las mayores posibilidades de intervenir ese fenómeno es en momentos previos o posteriores al desencadenamiento de las conductas violentas. A la escuela como institución social, le corresponde atender la socialización de las y los estudiantes, la integración de los valores, impulsar los sentimientos de pertenencia e identificaciones básicas. Para lograr el desarrollo de estos aspectos resulta fundamental que se cuente con procesos de prevención de la violencia, que procuren el fomento de una sana y pacífica convivencia en los centros escolares.

Martínez-Otero (2005) manifiesta que toda planificación educativa tendiente a solucionar la conflictividad escolar, debe tomar en cuenta el desarrollo personal y social de las personas que son parte de la comunidad estudiantil.

A continuación se presenta una serie de gráficos y cuadros que exponen los resultados de los datos obtenidos de los mecanismos empleados, por estudiantes y docentes, en la prevención de conductas violentas.

\section{Gráfico № 3}

Existencia de normas de convivencia durante los recreos de la escuela, en estudio. Año 2010.

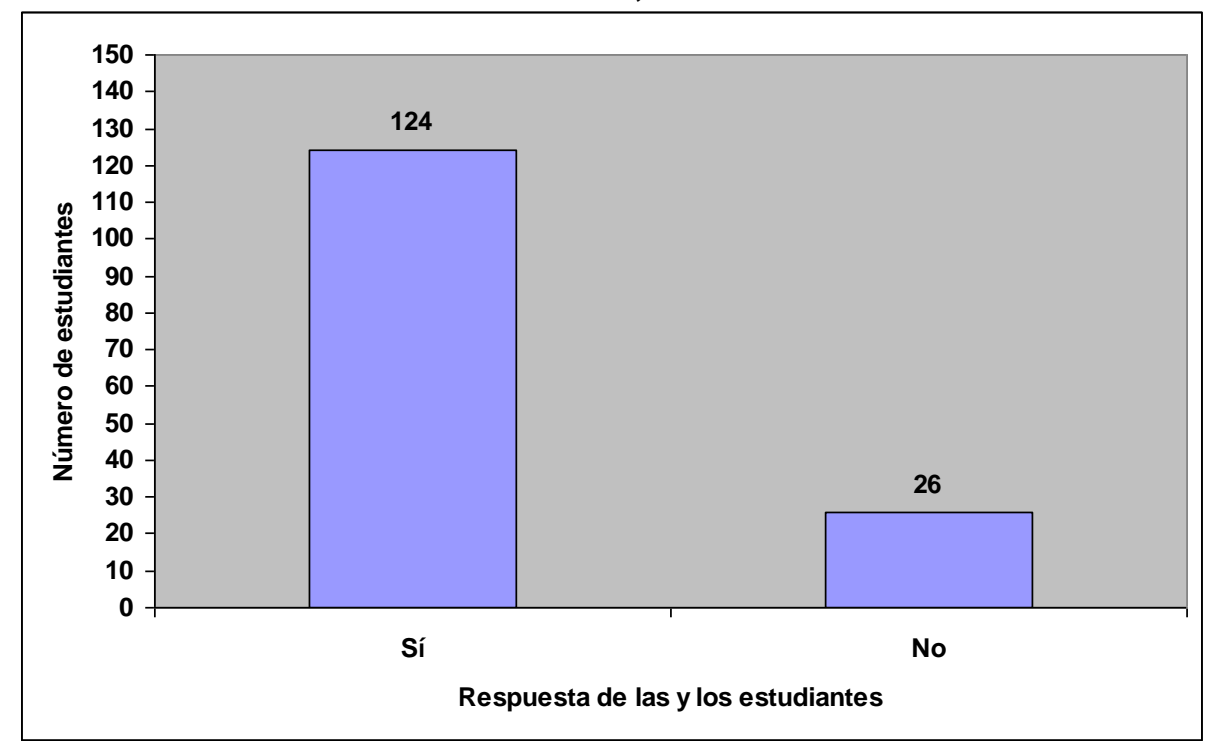

Fuente: Artavia, Jenny (2010). Resultados del cuestionario aplicado a estudiantes II Ciclo Enseñanza General Básica, escuela en estudio. 
El gráfico No 3 muestra información acerca de la existencia de normas de convivencia durante los recreos de la escuela, en estudio. Al respecto, se puede observar que 124 niños y niñas (82,6 por ciento) manifiestan que sí hay normas de convivencia y 26 estudiantes (17 por ciento) expresan que no existen normas de convivencia en la escuela. Al cuestionársele a la Directora acerca de la existencia de normas de convivencia en los recreos, ella señala que para los recreos propiamente no existen, que a las docentes les corresponde formar a las y los estudiantes en valores.

También indica la administradora, que existe legislación que hace referencia a los recreos, como son La Ley de Carrera Docente, El Código de Educación, La Ley de la Niñez y la Adolescencia. Asimismo, manifiesta que "la Ley de la Administración Pública, señala que somos depositarios del bien más preciado que son nuestros estudiantes, y se nos hace mención de que está legislado que los recreos le pertenecen a las niñas y niños y no a los docentes".

Al revisar la respuesta de la Directora ante esta interrogante, se reconoce que realmente no existen normas de convivencia para los recreos, como bien lo señalan los 26 estudiantes encuestados. Respecto a la respuesta positiva que dan las 124 niñas y niños restantes, se podría considerar que estos confunden quizás las normas de disciplina que el docente con su grupo, establece en cada sección.

Sería muy importante que la institución se dé a la tarea de implementar normas de convivencia consensuadas, que permitan la consecución de un adecuado clima escolar. Al respecto, San Martín (2003), enuncia que todo proceso educativo supone normas de convivencia que procuran alcanzar una educación cercana, preventiva, participativa y cooperativa. Señala el autor, que las y los educadores deben enseñar a sus estudiantes a crecer como personas, a crear hábitos de tolerancia, solidaridad, diálogo, los cuales permitan que el estudiante reciba una formación integral que les permita, el desarrollo de habilidades para la convivencia, para la gestión de conflictos y para el respeto de los derechos de los compañeros.

Desde la temática de las normas de convivencia, se les preguntó a las y los niños acerca de si se respetan las normas de convivencia en los recreos. Al respecto, 47 estudiantes (54,6 por ciento) opinaron que sí se respetan las normas de convivencia de los recreos. Por otra parte, 41 niños y niñas (64 por ciento) manifestaron que no se respetan 
esas normas y un estudiante opinó que no existen en la escuela normas de convivencia en los recreos.

El gráfico No 4 presenta la opinión de las y los estudiantes respecto a los mecanismos empleados por las y los docentes, para prevenir los conflictos. Como puede observarse, las y los niños señalan que existen varios mecanismos empleados por el personal docente en la prevención de los conflictos. La amenaza del envío de reportes es señalado por los chicos y las chicas como el mecanismo de mayor empleo en la escuela para tratar de prevenir los conflictos que se podrían presentar en la institución.

\section{Gráfico № 4}

Mecanismos empleados por las docentes para prevenir los conflictos, durante el desarrollo de los recreos de la escuela, en estudio. Año 2010

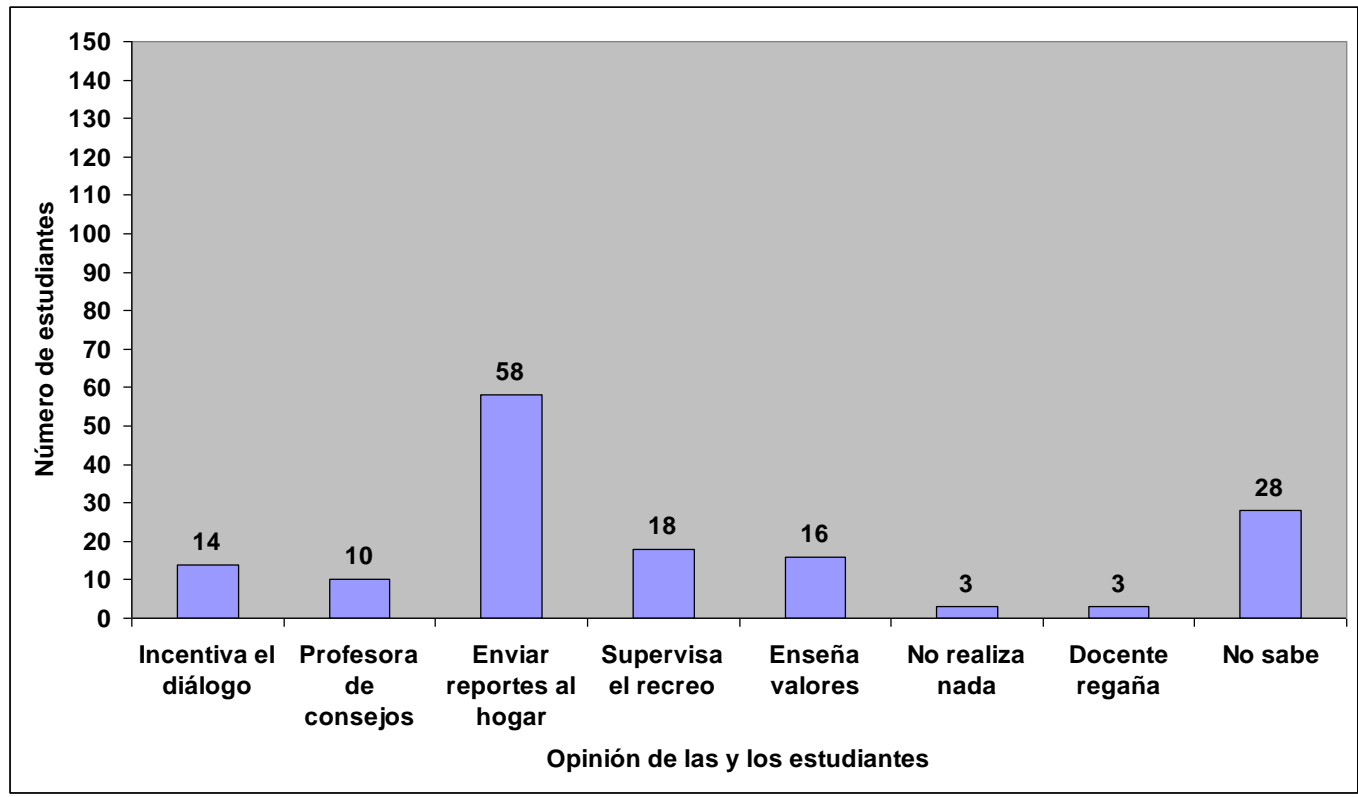

Fuente: Artavia, Jenny. (2010) Resultados del cuestionario aplicado a estudiantes II Ciclo Enseñanza General Básica, escuela en estudio.

En este gráfico se puede observar como 58 estudiantes (38,6 por ciento) hacen referencia que las y los docentes enfatizan en el envío de reportes al hogar, si hay conflictos durante los recreos. Asimismo, dieron respuesta a esta pregunta 28 de los sujetos del estudio (18,6 por ciento). En la misma manifiestan que no saben qué mecanismos se emplean, 18 niños y niñas (12 por ciento) opinan que las y los docentes supervisan los recreos, 16 (10,6 por ciento) señala que se les enseña valores como: el respeto y el trato 
hacia los demás. Catorce (9 por ciento) manifiesta que las docentes incentivan el diálogo entre las y los estudiantes; diez (7\%) aduce que les dan consejos; tres (2\%) que las docentes no hacen nada.

Desde esta perspectiva, se puede deducir que el procedimiento sancionador es el que prevalece en la institución para corregir conductas de indisciplina. Al respecto, San Martín (2003, p. 95) manifiesta que,

los procesos sancionadores, normalmente no resuelven los problemas, en general los agudizan. Las medidas coercitivas, en general, no encauzan ni educan. No se incide en la interioridad, en la raíz del problema, en las causas profundas, sólo se actúa sobre el exterior, restableciendo el orden conculcado, pero dejando la herida abierta.

Se citan a continuación algunos de las respuestas dadas por las y los niños, a la pregunta: ¿Cuáles son los mecanismos empleados por las docentes para prevenir los conflictos durante el desarrollo de los recreos?

\section{$\underline{\text { Respuestas estudiantes IV Año }}$}

- Nos dicen que no juguemos juegos bruscos.

- Amenazar con reportes o con mensajes.

- Estar vigilando y regañar o llamarle la atención al niño que causa daño.

\section{Respuestas estudiantes V Año}

- Diciendo que tengamos disciplina o mandando reportes.

- Decir que mandan reportes.

- Hablar con los compañeros.

\section{Respuestas estudiantes VI}

- Se sienta en los recreos y observa lo que pasa.

- Antes de salir nos dicen que nos comportemos y en los recreos nos vigilan.

- Algunos mecanismos que usa mi profesora, es darnos consejos a todo el grupo.

- Nos ayudan y nos enseñan varios valores.

- Nos dicen que nos pueden mandar reporte, nos pueden expulsar. 
Al analizar estas respuestas, se puede inferir que los mecanismos que las docentes emplean con mayor frecuencia, están muy apegados a procedimientos disciplinares ajustados a normas estrictas. En ellas predomina el uso de la sanción a través de boletas, amenazas con reportes, advertencias a las consecuencias y la vigilancia de los recreos por parte de las docentes.

También se observan en algunas de las respuestas dadas por las y los estudiantes, el reconocer que algunas de las docentes emplean otro tipo de procedimientos. Procuran la mediación del conflicto a través del diálogo, la reflexión, la formación en valores. Desde esta perspectiva se procura la resolución de conflictos de manera participativa y positiva, donde como bien lo señala San Martín (2003, p. 99), se procure:

- Promover la gestión positiva de los conflictos.

- Facilitar acuerdos constructivos.

- Pacificar las partes y reducir tensiones.

- Crear un ambiente escolar pacífico y constructivo donde se desarrolle: la afirmación personal y la autoestima, la confianza mutua, las capacidades de compartir sentimientos, información y experiencias y una actitud positiva y optimista ante la vida.

Resulta muy importante que los centros educativos, la familia y la sociedad en general, dediquen sus mayores esfuerzos en el desarrollo de una gestión constructiva del conflicto. Ahora bien, a la escuela le corresponde aprovechar el conflicto y la violencia, como oportunidades para el crecimiento personal y la convivencia y resolución pacífica de los mismos.

En el siguiente apartado se hace referencia a las estrategias que asume el centro escolar en estudio, para contrarrestar las manifestaciones de violencia presentes durante el desarrollo de los recreos.

\section{Estrategias empleadas por el personal docente y administrativo para contrarrestar las conductas violentas}

Ante el fenómeno de la violencia escolar es indispensable que todo centro educativo tenga organizado un modelo de convivencia escolar, que le permita implementar estrategias para contrarrestar las conductas violentas que se presenten. 
San Martín (2003), manifiesta que existen tres modelos de gestión de conflictos: el normativo (punitivo-sancionador), el relacional y el integrado. El modelo normativo se basa en la sanción o el castigo con la finalidad de que el estudiante no actúe de forma incorrecta o agresiva; el modelo relacional procura la comunicación directa entre las partes con el objetivo de que éstas se reconcilien; y el modelo integrador el cual integra los derechos y deberes de las personas a través de un proceso de mediación escolar.

Como parte de la investigación, se consideró pertinente indagar acerca de las estrategias que emplea el personal docente y administrativo de la escuela en estudio, para contrarrestar las conductas violentas. Los gráficos número cinco y seis presentan información relevante acerca de este tópico.

\section{Gráfico № 5}

Persona a la que acuden los niños cuando se dan conflictos en el recreo de la escuela, en estudio. Año 2010

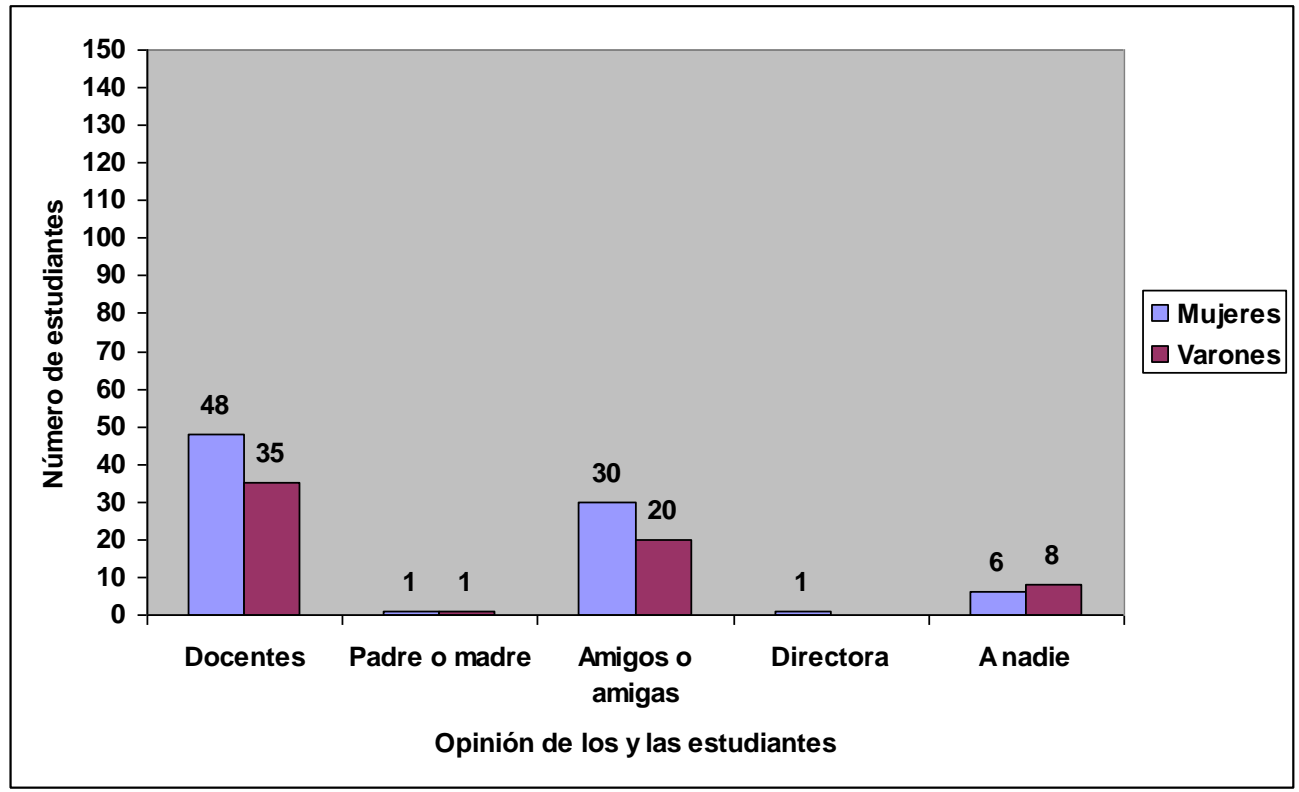

Fuente: Artavia, Jenny. (2010) Resultados del cuestionario aplicado a estudiantes II Ciclo Enseñanza General Básica, escuela en estudio.

El gráfico No 5 resume información concerniente a la opinión aportada por las y los niños ante la interrogante: ¿Cuando usted tiene conflictos en el recreo con alguien, a quién busca para que lo ayude? Al respecto se puede observar que la mayoría de las y los niños 
acuden a sus docentes para recibir ayuda por estos, cuando durante el desarrollo del recreo se presenta algún conflicto.

Obsérvese que en todos los casos, exceptuando la última opción (que no buscan a nadie para ser auxiliados), las niñas son las que en mayor número buscan ayuda en un adulto o en un par.

Otro gran porcentaje de niños (31 por ciento) y niñas (35 por ciento) buscan ayuda en amigos o amigas; seis mujeres ( 7 por ciento) y ocho varones (12,5 por ciento) opinan que no buscan a nadie cuando tienen conflictos durante los recreos. En el período de la preadolescencia, las y los niños reciben una gran influencia de sus pares; el introducirse en la vida del grupo resulta ser fundamental. La amistad responde a exigencias como son la defensa y la superación de dificultades en conjunto. $Y$, precisamente en un alto porcentaje las y los niños encuestados, responden que acuden a amigos o amigas en busca de ayuda para resolver los problemas de violencia o conflictos que se suscitan durante los recreos.

Ante la pregunta que se realizó a las y los estudiantes: ¿Qué acciones realiza la docente cuando se presenta algún problema de violencia en el recreo? La mayoría de las y los niños manifiestan que las docentes resuelven el problema de la violencia, enviando un reporte a los hogares, acción que como bien lo señala San Martín (2003), no corrige la conducta del agresor, pero sí produce resentimientos, rabia y si son injustos, tienden a no ser olvidados. Por otra parte, la víctima se siente sola, indefensa y sometida a una posible venganza. Por tanto, este tipo de modelo sancionador lleva a la confrontación y rompe la comunicación. Por otra parte, algunos niños y niñas señalan que sus docentes dialogan con ellos y procuran solucionar los problemas. Este modelo tal y como lo indica San Martín, procura prevenir el conflicto a través del diálogo, del encuentro interpersonal y parte "del principio de que reconciliarse con la víctima exige en el agresor un alto costo mental y emocional, lo cual supone un verdadero castigo para él. Además, este estilo de actuar sirve de modelo para que otros actúen de esta manera" (2003, p.45)

Al preguntar a las docentes: ¿Reciben quejas de niños o niñas por problemas de violencia durante los recreos? Las docentes externaron las siguientes opiniones:

- En algunas ocasiones, pero aisladas. Uno trata de de arreglar el problema en el momento, pero no muy frecuente. No llegan a diez las quejas en el año, en mi grupo. (Docente E de IV año, 22 de setiembre 2009). 
- Sí, que niña que me empujó, que niña que me golpeó, entonces casi siempre hay que Ilamar la atención. (Docente I de V año, 23 de setiembre 2009).

Es importante señalar cómo las y los estudiantes buscan en alguna medida, informar al adulto. En este caso, a las docentes, sobre las manifestaciones de violencia que se suscitan en los recreos, como producto de los juegos y de las relaciones interpersonales que se desarrollan entre los pares. Existe un nexo comunicativo de confianza y seguridad del niño hacia el adulto, ante las situaciones de violencia que se producen en el ámbito escolar.

Al consultar acerca de las estrategias empleadas por el personal docente y administrativo para contrarrestar las conductas violentas, se preguntó a las docentes y Directora: ¿Cuándo suceden problemas de violencia, qué mecanismos implementa la escuela para tratar de solventarlos?

Las docentes contestaron,

- Primero, se debe conversar con los niños a ver qué fue lo que sucedió. Si la violencia fue bastante, hay que aplicar algún proceso de acuerdo con el reglamento. (Docente $A$ de $V$ año, 23 de setiembre 2009)

- Confronto a los niños primero, luego busco a la docente del grupo si fuera necesario y si es mucho, aplico lo que dice el reglamento. (Docente T de IV año, 24 de setiembre 2009).

Al revisar las respuestas que dan las docentes, se infiere que existe una tendencia muy marcada al empleo de un modelo relacional en la resolución de las manifestaciones de violencia. Las docentes enuncian que una vez que se suscitó un problema de violencia entre las y los niños, en primera instancia acuden a implementar un proceso de comunicación entre las partes que procure su reconciliación. Si por el contrario, se percibe que la situación no se puede solucionar fácilmente 0 , dependiendo de la gravedad del problema, se procede a enviar el reporte como lo indica el reglamento.

Al consultar a la Directora, ella manifiesta que "la escuela puede aplicar el reglamento de conducta, donde define las partes especificadas con los números de puntos que les corresponden. Además, vienen las acciones correctivas". (26 de noviembre 2009) Considera que en la escuela se han dado a la tarea de poner en práctica acciones correctivas. Por ejemplo menciona que "este año se han puesto en práctica las acciones 
correctivas, así cuando se suscita algún problema se recurre a solicitarle al infractor, que prepare una pequeña charla sobre el tema que causó el problema. Agrega que esto es más significativo, que bajarles puntos y mandarlos para la casa".

Desde la perspectiva de los modelos para afrontar los problemas de violencia antes citados, la Dirección de la escuela impulsa un modelo normativo o sancionador mezclado con un modelo relacional. Se considera que se impulsa la aplicación de una sanción de forma oculta, donde el o los estudiantes deben preparar una charla sobre la temática que fue sancionada, para ser expuesta ante los demás compañeros. Con este tipo de acciones, se procura en primera instancia castigar a los agresores y víctimas, así como se previene a los demás estudiantes acerca de la situación que podrían estar enfrentando, si incurren en alguna falta, en la escuela.

\section{Nivel de integración del personal docente y administrativo de la escuela, durante el desarrollo de los recreos}

En este apartado se presenta información relacionada con el nivel de integración del personal docente y administrativo de la escuela en estudio. Básicamente con aspectos que tienen que ver con la supervisión durante el desarrollo de los recreos.

\section{Gráfico № 6}

Supervisión por parte de las y los docentes del desarrollo del recreo escolar, en la escuela en estudio. Año 2010

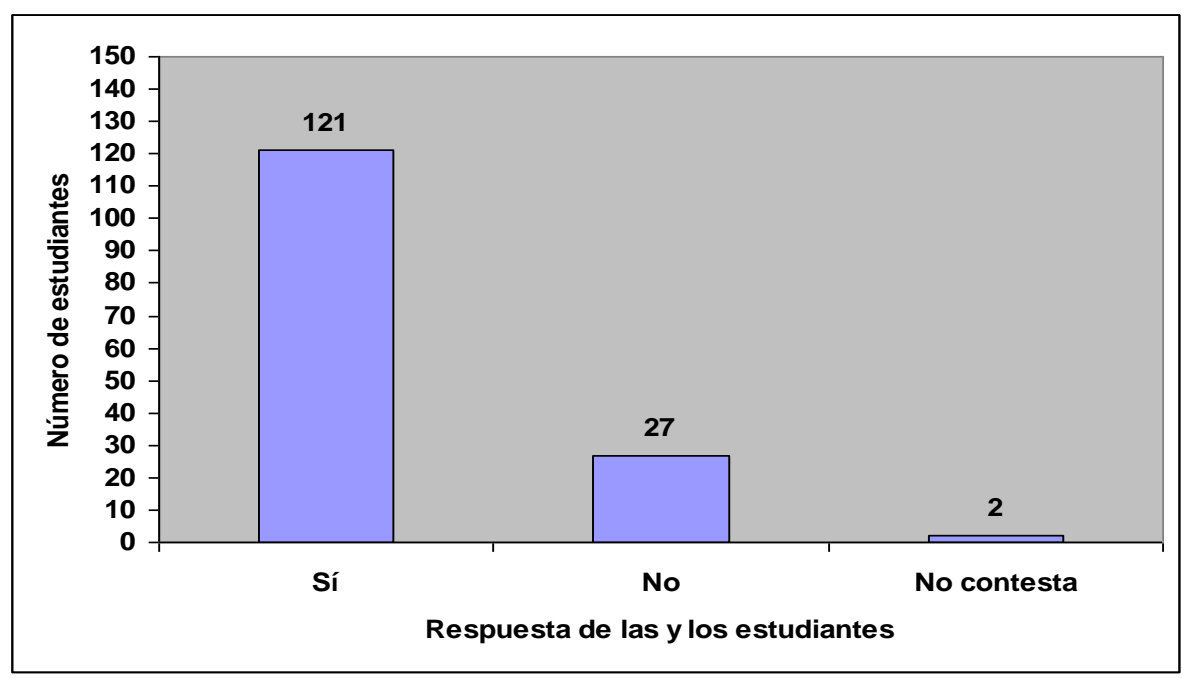

Fuente: Artavia, Jenny. (2010) Resultados cuestionario aplicado a estudiantes II Ciclo Enseñanza General Básica, escuela en estudio. 
El gráfico No 6 permite identificar las opiniones aportadas por las y los estudiantes respecto a si consideran importante que las y los docentes vigilen los recreos. Como bien puede observarse la mayoría de las y los niños, 121 de ellos (81 por ciento) considera que el personal docente sí debe supervisar el recreo, mientras que 27 (18 por ciento) opina que no deben vigilarlo.

Ahora bien, respecto a los motivos por los cuales sí deben vigilar el recreo, se presentan a continuación algunas de las opiniones dadas por las y los niños:

\section{Estudiantes de IV Año}

- Para parar las peleas y los problemas.

- Porque nos protegen.

- Porque después no hay testigos, si se pelea alguien.

- Así previenen accidentes.

\section{Estudiantes de V Año}

- Claro, para que no se den problemas.

- Para no golpearnos, pero a veces no nos cuidan porque están en reunión.

- Para que no se den conflictos.

- Porque si hay una pelea, ellos intervienen.

\section{Estudiantes de VI Año}

- Porque son la autoridad y así no suceden cosas graves.

- Para que no se den problemas y se evitan pleitos.

- Porque así los niños están más seguros.

- Para que los recreos sean más tranquilos.

Al revisar las respuestas de las y los estudiantes, se puede inferir que un alto porcentaje considera importante que el personal docente supervise el recreo. Obsérvese que en las respuestas dadas por la población estudiantil, la presencia de las docentes durante el recreo es percibida de forma positiva, pues para niñas y niños, el adulto representa seguridad, protección, alguien que está presto a intervenir en caso de que se suscite un conflicto entre estudiantes. 
Las docentes por su parte ante la pregunta: ¿Considera usted que es importante que los docentes supervisen los recreos? Opinan que,

- Sí, porque así podemos evitar diferentes accidentes. (Docente M de V año, 24 de setiembre 2009).

- Sí, es importante, porque si los docentes no están vigilando, ellos van a seguir más violentos. (Docente T de IV año, 24 de setiembre 2009).

- Sí para seguridad personal y que tengan buenas actitudes y mejoren como persona. (Docente A de V año, 23 de setiembre 2009).

En general se observa que existe anuencia por parte del personal docente a supervisar el recreo. Consideran que es importante y necesaria la presencia del docente en el desarrollo de los recreos, a través de procesos de supervisión de los juegos y demás actividades que desarrollan niños y niñas durante el recreo. Asimismo, expresan que necesitan también un espacio para ellas, para satisfacer sus necesidades personales y regresar a los salones de clase un poco más descansadas. Por su parte, la Directora manifiesta que,

Sí es muy importante. Soy partidaria de esto. Aquí el personal docente sabe que hay veces que no lo están haciendo y lo tienen por escrito. También tienen escritos las leyes y artículos, donde dicen que debemos cuidar los niños en el recreo y que el recreo es del estudiante, nunca del docente. (26 de noviembre 2009).

Durante el transcurso de las observaciones realizadas en el periodo de los recreos, se pudo constatar que cuando se inició el proceso de observación, la institución prácticamente no contaba con procesos de vigilancia por parte del personal docente y administrativo. Se hace referencia del término "prácticamente", porque en realidad el personal de la institución, la mayoría del tiempo dedicaba este tiempo a otras labores. Generalmente, la Directora siempre que tenía tiempo supervisaba el recreo y algunas veces dos o tres docentes realizaban esa labor. Sin embargo, la misión de supervisar el gimnasio recaía en el profesor de educación física. Y el pasillo que conduce al mismo, le era asignado a una conserje.

Se presenta a continuación algunos de los ejemplos de las acciones que realizan en la escuela en el proceso del recreo: 
- La Directora convocó a reunión de personal docente durante el desarrollo del recreo. (Observación realizada el día 22-9-08)

- Un día por la tarde llovió mucho. Los pasillos estaban muy mojados. Las y los niños corrían rápidamente y se mojaban en el patio. No había docentes que vigilaran los recreos. (Observación realizada el día 16-10-08)

- Varias docentes estaban conversando frente a la soda. otros estaban dialogando en la sala de profesores. Los niños y niñas corrían por los pasillos, con poca supervisión por parte del personal docente. (Observación realizada el día 17-10-08)

Al inicio del proceso de investigación, en la escuela en estudio los procesos de supervisión eran incipientes. Sin embargo, conforme la investigadora inició el proceso de observación de los recreos, la directora se dio a la tarea de organizar al personal docente para que supervisara a la población estudiantil durante el desarrollo de los recreos. Esta situación, tal vez se debió a la necesidad de brindar una respuesta en el ámbito educativo, ante los conflictos que se pudiesen generar entre estudiantes específicamente en el patio escolar durante el recreo escolar.

Anterior a esta nueva disposición emanada por la administradora, generalmente la Directora y al menos dos conserjes dedicaban algún tiempo a la supervisión del desarrollo de los recreos. El personal docente tomaba parte del tiempo destinado al recreo, para ingerir algún alimento, sacar fotocopias, conversar con sus colegas, entre otras actividades. También se pudo observar a algunas docentes más preocupadas por la supervisión del recreo. Así, entonces se pudo observar que:

- Una docente que estaba en la puerta de su aula, observaba lo que pasaba en el pasillo. Miraba que dos niños jugaban de forma muy agresiva el juego de la anda. Se acercó hasta donde estaban y les llamó la atención. (Observación realizada el día 29-9-08)

- Varias docentes estuvieron atentas de los juegos que los niños practicaban en los pasillos. En algunos momentos se dirigían hasta donde estaban algunos de ellos, y conversaron con ellos. (Observación realizada el día 6-10-08)

Al cuestionar a las docentes acerca de si, ¿supervisa usted el desarrollo de los recreos? Las docentes manifestaron: 
- Sí, porque está entre las tareas del docente cuidar los corredores y vigilar a los niños. (Docente D de VI año, 24 de setiembre 2009).

- Sí donde pueda. Digamos que sí, porque yo vigilo que no se golpeen, que no se suban a partes altas que son peligrosas, que no se den conductas inadecuadas. (Docente $T$ de IV año, 24 de setiembre de 2009).

Las respuestas dadas por las docentes, permite identificar que éstas tienen muy claro el papel de supervisión que se espera que lleven a cabo durante los recreos. Sin embargo, tal y como se indicó en las observaciones realizadas, este compromiso no fue evidente en la mayoría del personal. Generalmente el personal docente dedicaba este tiempo del receso escolar, para realizar otro tipo de actividades en espacios como el comedor, aulas, pasillos e inclusive la dirección.

La Directora considera que el personal docente debe estar atento a lo que ocurre en el transcurso del recreo entre las y los estudiantes. Opina que las docentes deben estar prestas a acoger las denuncias o quejas de las y los niños.

Martínez-Otero (2006) enuncia que una de las funciones que desempeña la cultura escolar es precisamente, la de regular las relaciones interpersonales y las actividades que se desarrollan, orientar el comportamiento de los miembros de la institución y controlar las acciones que se implementan en la institución.

\section{Conclusiones}

Esta investigación permitió llegar a las siguientes conclusiones:

- Un alto porcentaje de estudiantes manifiesta que existen enfrentamientos entre estudiantes durante el desarrollo de los recreos, y que estos enfrentamientos se visualizan a través de procesos de violencia tanto física como verbal.

- En los tipos de violencia explícita que se propician en las actividades lúdicas y en las interacciones personales de las y los estudiantes en los recreos, tienen predominio los juegos bruscos que propician violencia física y verbal. Las manifestaciones de violencia más comunes son los enfrentamientos, peleas, golpes y el uso de un lenguaje insultante y ofensivo.

- Respecto a la frecuencia con que se producen los enfrentamientos, se logró identificar que existe un mayor predominio de este tipo de violencia, entre las niñas. Las causas 
a las que se les atribuyen este tipo de conductas, son básicamente por rivalidades, envidias y celos entre las estudiantes.

- El motivo de los enfrentamientos varía según el nivel escolar. En el caso de las y los estudiantes de IV año los enfrentamientos se producen como resultado de los juegos que comparten. Por otra parte, en el caso de las y los niños de $\mathrm{V}$ y $\mathrm{VI}$ año los enfrentamientos obedecen a asuntos de diversa índole, entre ellos se citan: por la percepción que tienen del otro; viejos problemas personales ; o, por aspectos que tienen que ver con relaciones sentimentales que mantienen con compañeros del otro sexo. Por tanto, existe una marcada diferencia entre los motivos de los enfrentamientos que se suscitan entre estudiantes de IV año y los grados superiores de V y VI año, donde los procesos de desarrollo psicosocial de los preadolescentes podría estar marcando la diferencia entre estas poblaciones estudiantiles.

- Existe un alto nivel del empleo de violencia verbal. Se emplean malas palabras y expresiones despectivas y descalificadoras, que tienen como finalidad el ofender, humillar, insultar y molestar al otro.

- El personal docente y administrativo tiene conciencia de la problemática de la violencia física, verbal, psicológica y de intimidación, que se genera en la institución especialmente durante el desarrollo de los recreos.

- Respecto a los mecanismos de prevención de la violencia empleados por la escuela en estudio, se pudo constatar que las y los niños no tienen claridad acerca de la existencia de normas de convivencia para el desarrollo de los recreos. Asimismo, existe la concepción por parte de las y los estudiantes de que las normas de convivencia son elaboradas por otras personas, especialmente por los adultos, llámese docentes y Directora.

- La escuela no cuenta con normas de convivencia para el desarrollo de los recreos, ni tampoco con un plan de prevención y atención de la violencia. El personal docente y administrativo se rige únicamente por la aplicación de la legislación vigente.

- Los mecanismos empleados por la escuela para contrarrestar la violencia que se suscita en los recreos, se basa en un procedimiento sancionador. En este prevalece la aplicación de medidas correctivas. Existe un fuerte apego al uso de la sanción a través de boletas y reportes al hogar. En un menor porcentaje, se emplean procesos de 
resolución de conflictos más participativos. Algunas docentes emplean procedimientos de mediación del conflicto a través del diálogo, la reflexión y la formación en valores.

- La Dirección de la escuela impulsa la aplicación de acciones donde el estudiante que violenta a otro, debe organizar una charla sobre la temática que fue sancionado y exponerla ante el resto de compañeros. Este tipo de acciones obedece a un modelo de sanción que se implementa de forma oculta, donde el estudiante agresor recibe su castigo y sirve como proceso de prevención con el resto de estudiantes.

- Respecto al nivel de integración del personal docente y administrativo en el desarrollo de los recreos, existe un alto porcentaje de estudiantes que consideran importante que las y los docentes vigilen los recreos. El proceso de vigilancia representa para las y los niños, seguridad, protección y asistencia pronta de un adulto, en caso de que ocurra algún conflicto entre estudiantes.

- El personal docente en general considera importante la supervisión del recreo. Sin embargo, en las observaciones realizadas se pudo constatar que un alto porcentaje del personal docente, no está totalmente comprometido con el proceso de supervisión del recreo.

\section{Recomendaciones}

\section{Al Ministerio de Educación Pública}

- Capacitar al personal docente y administrativo de las Direcciones Regionales, acerca de los procesos de prevención e intervención para la atención de la violencia escolar.

- Capacitar al personal docente y administrativo en los procedimientos legales que deben seguir los centros educativos para cumplir con lo estipulado en el Código de la Niñez y la Adolescencia.

- Mayor supervisión de la Dirección de infraestructura y equipamiento educativo (MEP), para hacer cumplir la reglamentación relacionada con el uso de los espacios físicos. Especialmente, aquellos espacios sin techo destinados al juego y la recreación de las y los estudiantes.

- Fomentar procesos de investigación en las regiones, que permitan analizar de forma integral el fenómeno de la violencia que se desarrolla en las instituciones educativas. 


\section{A la Dirección Regional de Educación Occidente}

- Impulsar procesos de capacitación al personal docente y administrativo de las instituciones educativas de la región, acerca de los procesos de prevención e intervención para la atención de la violencia escolar.

- Capacitar al personal docente y administrativo en los procedimientos legales que deben seguir los centros educativos para cumplir con lo estipulado en el Código de la Niñez y la Adolescencia.

- Impulsar alianzas estratégicas con la Sede de Occidente de la Universidad de Costa Rica, que permitan el desarrollo de procesos de investigación y de acción social en pro de la prevención de la violencia.

\section{A la Sede de Occidente de la Universidad de Costa Rica}

- Impulsar procesos de investigación y acción social (Trabajo Comunal Universitario, Extensión Docente) que permitan desarrollar procesos integrales, en pro de la prevención de la violencia de la y en las instituciones educativas de la Región de Occidente.

\section{A la escuela en estudio}

- Fomentar procesos de capacitación al personal docente, acerca de los procesos de prevención e intervención para la atención de la violencia que se pueda presentar en la escuela.

- Elaborar normas de convivencia del centro educativo que procuren el diálogo y un eficiente tratamiento de los conflictos, en forma constructiva, de modo conjunto entre estudiantes, personal docente y personal administrativo.

- Mejorar los espacios físicos en cantidad y calidad, destinados al juego y la recreación de la población estudiantil.

\section{Referencias}

Abad, Susana. (2002). Acerca de las conductas violentas en el aula. Cuando la asimetría lleva a la humillación. Ensayos y experiencias, (44), 30-45.

Abramovay, Miriam. (2005). Violencia en las escuelas: un gran desafío. Revista Iberoamericana de Educación II, (38), 13-26. 
Artavia, Granados, Jenny. (2010). La violencia escolar explícita e implícita en las y los estudiantes de segundo Ciclo de la Enseñanza General Básica de una escuela del circuito 01 de la Dirección Regional de Educación Occidente, durante el desarrollo del recreo. Informe final de investigación inédita, No. 540-A8-305, San Ramón, Costa Rica, Universidad de Costa Rica.

Brawer, Mara. (2006). Hacia la construcción del conocimiento que oriente el diseño de políticas públicas. Violencia en las escuelas. Recuperado de http://www.emprendimientos.org.ar/index archivos/3nota8.pdf

Casals, Judith. (2006). ¿Qué nos dicen las encuestas y los estudios?: Cuadernos de pedagogía, (359), 44-51.

Martínez-Otero, Valentín. (2005) Conflictividad escolar y fomento de la convivencia. Revista Iberoamericana de Educación, (38), 89-107.

Martínez-Otero, Valentín. (2006). Comunidad educativa. Claves psicológicas, pedagógicas y sociales. Madrid: Editorial CCS.

Nogueira, Rosana María. (2005). A prática de violência entre pares: o bullying escolas. Revista Iberoamericana de Educación, (37), 93-102.

Organización Mundial de la Salud. (2001). Informe mundial sobre la violencia y la salud. Ginebra: O.M.S. Recuperado de www.who.int/violence-injuryprevention/violence/word-report/en/abstract-es.pdf

Ortega, Rosario. (2004). Víctimas, agresores y espectadores alumnos implicados en situaciones de violencia. Recuperado de: http://www.hika.net/zenb160/H16024.HTM

Osorio, Fernando. (2006). Violencia en las escuelas. Un análisis desde la subjetividad. Buenos Aires: Centro de Publicaciones Educativas y Material Didáctico.

San Martín, José Antonio. (2003). La mediación escolar. Un camino nuevo para la gestión del conflicto escolar. Madrid: Editorial CCS.

Vinyamata, Eduard. (2003). Aprender del conflicto. Conflictología y educación. Barcelona: Graó. 